\title{
Vers une utilisation de données probantes en oncologie: problèmes rencontrés et solutions suggérées
}

par Nicole Allard et Christina Jalbert

\section{Abrégé}

La récente préoccupation des milieux cliniques à utiliser des pratiques exemplaires issues de données probantes est grandissante. Nous voyons apparaître différentes formations et ressources pouvant être utilisées par les infirmières en oncologie. Cette étude met en relief, à l'aide d'un devis à séries temporelles, qu'après une intervention sous forme d'une présentation formelle PowerPoint et de cartables distribués aux infirmières sur les données probantes, l'utilisation véritable des données probantes dans la pratique est inchangée. Il en ressort que très peu d'infirmières s'y intéressent et que celles qui les utilisent sont celles qui le font dans le cadre de leurs fonctions (cadre conseil, cliniciennes). Des recommandations pour la pratique, la formation et la recherche sont suggérées.

Le Canada connaîtra une véritable recrudescence du cancer d'ici quelques années. Effectivement, avec le vieillissement des babyboomers et l'augmentation globale de la population canadienne, le cancer deviendra la principale cause de mortalité d'ici 2010. Ainsi, les nouvelles statistiques canadiennes sur le cancer (Société canadienne du cancer, 2010) estiment qu'en 2010, au Québec, il y aura 45200 nouveaux cas de cancer (173800 au Canada) et 20300 décès attribuables à cette maladie (76200 au Canada). D'après les taux d'incidence actuels, près de $40 \%$ des Canadiennes et de $45 \%$ des Canadiens seront atteints d'un cancer au cours de leur vie. Le cancer le plus fréquemment diagnostiqué demeure le cancer du sein chez la femme et celui de la prostate chez l'homme. Cependant, la principale cause de décès associée au cancer autant chez les femmes que chez les hommes continue d'être le cancer du poumon (Santé Canada, 2009; Société canadienne du cancer, 2010).

En plus d'être une épreuve personnelle très difficile à vivre, le cancer entraîne des effets dévastateurs pour la santé économique de notre pays. On estime qu'au cours des 30 prochaines années, près de 6 millions de Canadiens auront reçu un diagnostic de cancer; près de 3 millions de Canadiens mourront des suites de la maladie; les coûts de santé directs associés au cancer dépasseront les 39 milliards et l'incapacité liée au cancer entraînera la perte de plus de 101 milliards en recettes fiscales. En conséquence, le défi social est d'envergure puisqu'il engendre la maladie sur le plan humain et économique. Des répercussions considérables sont ressenties en matière de soins de santé et son incidence s'accroît

\section{Au sujet des auteures}

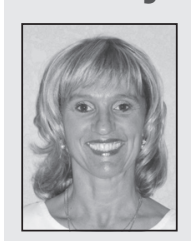

Nicole Allard, inf., Ph.D., Professeure, Co-directrice module des sciences de la santé, Département des sciences infirmières, Université du Québec à Rimouski, Campus de Lévis, Bureau 3063, 1595 boulevard Alphonse Desjardins, Lévis, Québec G6V 0A6. 418-833-8800 poste 3274; Nicole_allard@uqar.qc.ca

Christina Jalbert, Infirmière à l'Hôtel-Dieu de

Lévis (Pédopsychiatrie), 143 rue Wolfe, Lévis, Québec G6V $3 Z 1$. 418-835-7121 poste 3037; christina.jalbert@hotmail.com davantage à cause du vieillissement de la population. De plus, le Québec présente le fardeau économique de soins de santé le plus lourd des provinces canadiennes ainsi que dans la majorité des pays de l'Organisation de coopération et de développement économiques (OCDE) (Duhamel, 2006).

Les personnes atteintes de cancer, tout comme leur entourage, ont besoin d'information sur la façon d'évaluer et de gérer leurs symptômes en période aiguë et surtout en postopératoire, d'autant plus qu'elles retournent souvent à la maison le jour même de l'intervention. C'est d'ailleurs ce constat qui incitait le gouvernement à mettre sur pied en 1998 le Programme québécois de lutte contre le cancer (PQLC). Celui-ci vise à concerter les efforts afin que les habitudes de vie soutiennent la prévention, le dépistage précoce, l'amélioration et le maintien de l'évolution de la qualité des services d'investigation et des traitements, de même que l'accessibilité des soins, plus particulièrement des soins palliatifs (Comité consultatif sur le cancer, 1998). Le PQLC accorde aussi une attention particulière à la continuité des services ainsi qu'au soutien apporté tout au long de la trajectoire de la maladie.

Selon Sackett, Rosenberg, Gray, Haynes et Richardson, la pratique de soins basée sur des données probantes est «l'utilisation consciencieuse et judicieuse des meilleures données probantes récentes dans la prise de décisions concernant les soins aux patients individuels. Elle signifie l'intégration de l'expertise clinique personnelle aux meilleures données probantes qui soient, tirée de recherches systématiques» (Sackett, Rosenberg, Gray, Haynes \& Richardson, 1996). De fait, l'Oncology Nursing Society des États-Unis offre maintenant en ligne les dernières données probantes et donne un accès aux articles de recherche afin de promouvoir une pratique infirmière basée sur des résultats probants. Ce site Web ainsi que d'autres ressources disponibles présentent les lignes directrices récentes afin de soulager plusieurs symptômes répertoriés tels que la fatigue, la douleur, la nausée et les vomissements, l'anorexie, la neuropathie périphérique, le lymphœdème, la dépression et l'anxiété (Oncology Nursing Society, 2009b, 2010).

Les personnes atteintes de cancer et leurs proches ont assurément besoin de soutien émotionnel. Le soutien est considéré comme essentiel à l'efficacité de l'ensemble des interventions destinées aux personnes atteintes de cancer (Comité consultatif sur le cancer, 1998). D'ailleurs, selon l'Association canadienne des infirmières en oncologie, le respect des normes de pratique en oncologie et le travail des équipes fonctionnant en interdisciplinarité représentent des éléments importants dans le suivi et le soutien offerts (2006). Alors, les protocoles de soins cliniques et les lignes directrices basées sur des résultats empiriques probants sont les progressions les plus prometteuses et efficaces pour définir et améliorer la qualité des soins auprès de la clientèle atteinte de cancer (Miller \& Kearney, 2004). L'intervenant pivot en oncologie, par exemple, représente un changement positif dans le but de répondre aux besoins de continuité et de concertation dans les soins exprimés par les personnes atteintes de cancer (de Serres \& Beauchesne, 2000). Ceci dit, il importe de considérer les symptômes physiques, la détresse émotionnelle et l'adaptation à la maladie chez les personnes atteintes de cancer et non seulement les indicateurs financiers, de morbidité et de satisfaction. 
Les infirmières et les intervenants en oncologie doivent quotidiennement faire face à des situations dans lesquelles leurs connaissances théoriques et pratiques sont nécessaires en vue de soigner des symptômes physiques et psychologiques. C'est pourquoi il s'agit de leur offrir les résultats les plus probants sur le plan scientifique qui guideront leur pratique professionnelle puisque ces professionnels n'ont ni le temps requis ni assez de compétences pour les traiter (Association canadienne des infirmières en oncologie, 2006). Les lignes directrices doivent contenir des renseignements appropriés et exacts pour les patients, fondés dans la mesure du possible sur la recherche et des sources de références reconnues. Selon les dernières données, les interventions éducatives de soutien et de résolution de problèmes sont les plus indiquées pour aider les personnes atteintes de cancer (Comité consultatif sur le cancer, 1998). Les études de Leventhal et Johnson (1983) ont fourni une base théorique fondée sur la théorie de l'autorégulation ainsi qu'un support empirique pour préparer les patients à la chirurgie, aux procédures médicales stressantes et à la radiothérapie. D'autres chercheurs ont également examiné l'efficacité d'une intervention psychoéducative infirmière et ont démontré que ce type d'information améliore le retour au fonctionnement habituel dans les activités quotidiennes après une chirurgie (Hill, 1982; Moore, 1996), réduit la détresse associée à des procédures médicales stressantes et à la douleur (Suls \& Wan, 1989) et aide les patients atteints de VIH à mieux gérer et soulager leurs symptômes dans une perspective d'autorégulation (Côté \& Pepler, 1999; Côté \& Pepler, 2002). Une étude clinique récente (Allard, 2005) suggère également qu'une intervention infirmière téléphonique psychoéducative basée sur la théorie de l'autorégulation et le concept de redirection est efficace pour rehausser le niveau de fonctionnement et réduire la détresse émotionnelle chez les femmes atteintes de cancer du sein durant les 3 premières semaines suivant la chirurgie.

Malgré le nombre croissant de documents et d'études rigoureuses disponibles documentant les résultats de recherche et la façon optimale de gérer les symptômes des personnes atteintes de cancer, peu d'interventions ou de suivis sont effectivement implantés dans les milieux cliniques. Malheureusement, la non-utilisation des résultats de recherche engendre plusieurs désavantages autant pour le personnel infirmier que pour les patients et leur famille. Avec le développement rapide des services de santé, des défis majeurs s'annoncent pour tous les professionnels. Effectivement, ceux-ci doivent parfaire les techniques de soins pour offrir les meilleurs services possibles à la population (Halabis-Nassif \& Hatem, 2008). Or, la pratique en soins infirmiers et la prise de décision des infirmières ont tendance à être fondées sur la propre expérience clinique des intervenants en oncologie, sur des infirmières d'expérience ou sur la tradition (Egerod \& Hansen, 2005; Estabrooks, Floyd, Scott-Findlay, O'Leary \& Gushta, 2003). En ne s'actualisant pas par rapport aux dernières données probantes, la profession infirmière et les infirmières qui la composent stagnent et ne maintiennent pas les standards de qualité attendus. Donc, il en découle directement une diminution de la qualité des soins et de la satisfaction des patients qui utilisent les services de santé (Halabis-Nassif \& Hatem, 2008).

\section{Cadre théorique}

Dans le but de mesurer, de disséminer et d'utiliser des données probantes en pratique, le cadre théorique d'Estabrooks (1999) a été utilisé. Cette dernière a opérationnalisé la notion d'utilisation globale de données probantes (overall research utilization) dans la pratique infirmière selon 3 concepts distincts: l'utilisation directe, indirecte et persuasive. Ce cadre théorique, ces concepts ainsi que les mesures utilisées par Estabrooks ont été utilisés afin d'évaluer le transfert des connaissances dans la pratique infirmière en oncologie, avant et après la présentation sur les données probantes afin qu'elles les connaissent et les utilisent. L'utilisation instrumentale est recherchée. Il s'agit de l'application concrète de la recherche et celle-ci est normalement traduite dans un format utilisable, tel qu'un protocole (Estabrooks, 1999). Une étape importante de la pratique fondée sur des résultats probants est celle de la diffusion des résultats. La diffusion formelle a l'avantage de diffuser à un auditoire plus large et diversifié et d'augmenter la probabilité d'un changement dans les milieux de soins (Goulet, Lampron, Morin \& Heon, 2004).

\section{Méthodologie}

Un devis à séries temporelles a été utilisé: il s'agit d'une étude avec de nombreuses prises de mesures de l'utilisation de résultats probants dans la pratique avant et après l'intervention, auprès d'un seul groupe et à des moments précis. Les effets de la variable s'évaluent par l'observation d'une discontinuité dans la série plutôt que par la comparaison avec un autre groupe. Ce devis requiert peu de participants (Fortin, 2010).

L'intervention consistait en une présentation formelle (PowerPoint lors de midis cliniques) de résultats probants de gestion des symptômes en oncologie et de distribution de cartables de références de ces résultats probants (Oncology Nursing Society, 2009a, 2010) sur chacune des unités concernées (chimiothérapie, radiothérapie, clinique de chirurgie). L'observation 01 représente la période de collecte des données (soins usuels) avant l'intervention et les observations 02 et 03 correspondent à deux périodes de collecte des données après l'intervention. L'observation 02 s'est effectuée environ 1 mois après la première collecte des données afin de mesurer l'impact à court terme de l'intervention et l'observation 03 s'est effectuée environ 6 mois après, afin de vérifier le niveau de rétention du personnel infirmier participant.

\section{Participants}

L'échantillon de convenance final était constitué de 1 homme et de 17 femmes exerçant la profession infirmière. Ces derniers devaient faire partie de l'Ordre des infirmières et infirmiers du Québec et exercer en oncologie pour participer à cette étude.

\section{Sites}

Les centres dans lesquels ont été recrutés les participants sont deux centres régionaux et un centre urbain au Québec. Tous ces milieux offraient des services et des traitements aux personnes atteintes de cancer.

\section{Instruments}

Un questionnaire sociodémographique conçu pour cette étude (incluant l'âge, le sexe, le niveau d'éducation, le nombre d'années d'expérience à titre d'infirmière et la fonction clinique dans l'établissement) a d'abord été rempli par les participants.

Par la suite, le questionnaire d'utilisation des données probantes dans la pratique développé par Estabrooks (1999) (version anglaise originale) a été choisi et traduit car il comporte l'avantage d'être très court à utiliser. Ce questionnaire inclut 3 questions mesurant l'utilisation globale des résultats probants de recherche: l'utilisation directe, indirecte et persuasive. Les réponses aux questions sont codées selon ces critères: $1=$ jamais, $2=$ un ou deux quarts de travail, $3=($ non codée), $4=($ non codée), 5=environ la moitié des quarts de travail, $6=$ (non codée), $7=$ presque tous les quarts de travail et $8=$ je ne sais pas. Le modèle retenu représentant la structure conceptuelle de l'utilisation de données probantes démontre un haut niveau de validité de construit (goodness of fit R2) élevé, près de $70 \%$ de la variance de la variable dépendante est expliquée par ce modèle. La consistance interne de l'instrument varie de 0,77 à 0,91 pour les sous-échelles $(n=600)$ (Frasure, 2008). Il a été traduit en français et validé par des experts bilingues en oncologie selon la méthode inversée de Vallerand (1989). 


\section{Consentement}

Le consentement des infirmières en oncologie qui ont participé à ce projet pilote a été sollicité avant la présentation formelle sur l'utilisation des données probantes lors des ateliers durant le dîner (annexe 1). Elles ont complété un bref questionnaire sociodémographique, ainsi que le questionnaire d'utilisation globale des résultats probants de recherche (temps 01).

\section{Confidentialité}

Toutes les données de l'étude ont été placées dans un classeur, sous clé. Seul le chercheur principal ainsi que les assistantes de recherche ont accès à ces données confidentielles. Afin que l'on ne puisse identifier les participants, le dossier de recherche est identifié à l'aide d'un code et le nom n'y apparaît pas. Toutes publications ou communications scientifiques provenant de cette recherche sont rédigées de façon à ce qu'il soit impossible de retracer la personne.

\section{Déroulement du projet}

Les infirmières en oncologie (dont les infirmières pivots) ont été sollicitées et impliquées tout au long de cette recherche. Une publicité dans chacun des centres a été distribuée pour inviter les

\begin{tabular}{|l|c|}
\hline \multicolumn{2}{|l|}{$\begin{array}{l}\text { Tableau 1. Alpha de Cronbach des sous-échelles du } \\
\text { questionnaire traduit des données probantes }\end{array}$} \\
\hline Sous-échelles & Alpha de Cronbach \\
\hline Utilisation directe & 0,61 \\
\hline Utilisation indirecte & 0,71 \\
\hline Utilisation persuasive & 0,83 \\
\hline Total & 0,82 \\
\hline
\end{tabular}

Tableau 2. Données sociodémographiques

\section{Formation scolaire}

\begin{tabular}{|l|c|}
\hline Cégep & $1(5,6 \%)$ \\
\hline Certificat & $2(11,1 \%)$ \\
\hline Université & $9(50 \%)$ \\
\hline Maîtrise & $4(22,2 \%)$ \\
\hline Manquant & $2(11,1 \%)$ \\
\hline
\end{tabular}

\section{Spécialisation}

\begin{tabular}{|l|c|}
\hline Oui & $10(55,6 \%)$ \\
\hline Non & $6(33,3 \%)$ \\
\hline Manquant & $2(11,1 \%)$ \\
\hline
\end{tabular}

\section{Fonction}

\begin{tabular}{|l|c|}
\hline Infirmière bachelière & $5(27,8 \%)$ \\
\hline Infirmière pivot & $1(5,6 \%)$ \\
\hline Infirmière chef & $3(16,7 \%)$ \\
\hline Cadre conseil & $1(5,6 \%)$ \\
\hline Infirmière technicienne & $3(16,7 \%)$ \\
\hline Enseignant & $1(5,6 \%)$ \\
\hline BACC & $1(5,6 \%)$ \\
\hline Manquant & $2(11,1 \%)$ \\
\hline Clinicienne & $1(5,6 \%)$ \\
\hline
\end{tabular}

cliniciens en oncologie à assister à une présentation sur l'utilisation des données probantes en oncologie. Cette présentation sur les données probantes d'une durée approximative d'une heure a été présentée dans chacun des centres. Par la suite, les infirmières désirant participer à la recherche ont été informées du but et du déroulement de la recherche et ont signé un formulaire de consentement éclairé. Des cartables contenant des définitions, les facteurs de risque, de nombreuses références et instruments de mesure sur les principales données probantes en regard de la gestion des principaux symptômes en oncologie ont été édifiés à partir de données probantes les plus récentes provenant de l'Oncology Nursing Society. Ces symptômes incluaient: douleur, neuropathie, fatigue, insomnie, niveau de fonctionnement, dépression, intervention téléphonique, mucosite, nausées et vomissements (Updated Oncology Nursing Society putting evidence into practice resources, 2009). Ces cartables ont été fournis pour les participants travaillant dans chacune des unités de soins participant à l'étude. Ensuite, les collectes des données 02 et 03 ont été effectuées par téléphone par l'assistant de recherche 1 mois et 6 mois après l'intervention (la présentation sur les données probantes). Ces collectes avaient pour but de déterminer si l'utilisation des données probantes avait changé chez les participants après la présentation et la fourniture des cartables contenant les données probantes pour le soulagement de symptômes physiques et psychologiques.

\section{Analyses effectuées}

Le degré d'homogénéité de l'instrument sur l'utilisation des données probantes a été vérifié à l'aide du coefficient alpha de Cronbach. Alors, les résultats ont démontré un coefficient de 0,82 pour l'instrument global (incluant toutes les sous-échelles), indiquant une bonne consistance interne. De plus, les coefficients alpha de Cronbach ont été mesurés pour chacune des sous-échelles de l'instrument (tableau 1).

Cela signifie que la cohérence interne est bonne pour le score global et celui de l'utilisation persuasive, car un coefficient supérieur à 0,80 est considéré acceptable pour un instrument de recherche (Burns \& Grove, 2007, 2009). Les coefficients de Cronbach pour les sous-échelles de l'utilisation directe et indirecte n'ont cependant pas atteint ce seuil. Par la suite, des analyses descriptives telles que des moyennes et des écart-types ont été calculées afin de connaître la répartition des sujets selon les données sociodémographiques de chaque participant et selon l'utilisation des données probantes. De plus, des corrélations de Pearson ont été effectuées pour observer s'il y a un lien entre les trois collectes de données. Finalement, des analyses de fonction discriminante pas-à-pas ont été réalisées pour discerner quelles variables sociodémographiques prédisaient le mieux le niveau d'utilisation des données probantes.

\section{Résultats}

\section{Caractéristiques de l'échantillon}

$\mathrm{Au}$ cours de la collecte de données qui s'est déroulée sur une période de 6 mois, 18 participants ont été recrutés soit 1 homme $(5,6 \%)$ et 17 femmes $(94,4 \%)$. La répartition des sujets selon les données sociodémographiques est présentée au tableau 2.

\section{Résultats face à l'utilisation des données probantes}

Tout d'abord, des moyennes ont été calculées pour chacun des scores des sous-échelles et du score total pour chacun des trois temps de collecte de données (tableau 3).

Ensuite, des corrélations ont été effectuées entre les trois temps de collecte de données (tableau 4). Premièrement, la corrélation entre la somme des temps 01 et 02 s'est révélée significative face à l'utilisation des données probantes avec un coefficient de $\mathrm{R}=0,86$. Deuxièmement, la corrélation entre la somme au temps 01 et 03 a obtenu un résultat non significatif de $\mathrm{R}=0,44$. Troisièmement, la 
corrélation entre la somme au temps 02 et 03 est de $\mathrm{R}=0,35$, ce qui n'est pas utile pour prédire l'utilisation des données entre ces deux temps.

Des régressions multiples de type pas-à-pas ont été effectuées sur les résultats obtenus afin de vérifier quelle variable permettait de mieux prédire l'utilisation des données probantes. La marge d'erreur des donnés est de 0,05\%. Tout d'abord, au temps 01, la formation scolaire est significative pour prédire l'utilisation directe des données probantes dans le milieu avec une variance de $\mathrm{R}=0,54$. Aucun élément sociodémographique n'est statistiquement significatif pour prédire l'utilisation indirecte. Somme toute, la spécialisation serait un élément clé pour prévoir l'utilisation persuasive des données probantes au temps 01 avec une variance de $\mathrm{R}=0,567$. Pour le temps 02, aucun élément sociodémographique ne permet de supposer l'utilisation directe et indirecte des données probantes. Par contre, la spécialisation permet d'obtenir une variance de $\mathrm{R}=0,686$, ce qui est significatif afin de prédire l'utilisation persuasive au temps 02. De plus, la formation scolaire conjuguée à la spécialisation augmente les probabilités d'utiliser de façon persuasive les donnée probantes avec une variable de $\mathrm{R}=0,790$. Finalement, aucun élément sociodémographique ne permet de prévoir l'utilisation directe et indirecte au temps 03. Pour ce qui est de l'utilisation persuasive au temps 03, les années d'expérience seraient un facteur facilitateur avec une variance de $\mathrm{R}=0,596$. Pour finir, des analyses de type Anova à plan simple ont été effectuées. Aucune ne s'est révélée significative entre les temps 01,02 et 03, et ce, pour l'ensemble des sous-échelles.

\section{Discussion et recommandations}

\section{Pratique}

Nous avons effectué une intervention d'enseignement sur les données probantes en milieu clinique. Les participants à l'étude ont reçu une formation sur les données probantes d'une durée de 1 heure. À la suite de la formation, un cartable a été remis aux participants afin qu'ils puissent consulter facilement les données probantes de recherche provenant de l'Oncology Nursing Society (Adams et al., 2009) sur chacun des symptômes les plus fréquemment mentionnés par les patients. Les résultats de notre étude ont été obtenus par le biais d'un questionnaire que les participants remplissaient à trois reprises selon le protocole établi. Les auteures ont été surprises des résultats obtenus lors des analyses descriptives telles que la moyenne et l'écart-type. Nous constatons que les moyennes sont sensiblement les mêmes au temps 01, 02 et 03. Nous pouvons donc affirmer que l'intervention d'enseignement sur les données probantes n'a pas produit les résultats escomptés. Aucune augmentation de l'utilisation des données probantes n'est survenue au cours des six mois de l'étude. Les auteures de l'étude se sont questionnées sur les facteurs ayant pu influencer les résultats.

Tout d'abord, le recrutement des participants dans les trois sites s'est effectué sur une base volontaire. Il est probable que

\begin{tabular}{|l|l|l|l|c|}
\hline \multicolumn{6}{|l|}{$\begin{array}{l}\text { Tableau 3. Description des moyennes } \\
\text { d'utilisation des données probantes }\end{array}$} \\
\hline $\begin{array}{l}\text { Étendue: } \\
\text { 1-7 }\end{array}$ & $\begin{array}{c}\text { Utilisation } \\
\text { directe }\end{array}$ & $\begin{array}{c}\text { Utilisation } \\
\text { indirecte }\end{array}$ & $\begin{array}{c}\text { Utilisation } \\
\text { persuasive }\end{array}$ & $\begin{array}{c}\text { Moyenne de } \\
\text { la somme des } \\
\text { temps }\end{array}$ \\
\hline $\begin{array}{l}\text { Moyenne au } \\
\text { temps 01 }\end{array}$ & $4,19(1,68)$ & $5,17(1,62)$ & $4,44(1,85)$ & $4,5(1,40)$ \\
\hline $\begin{array}{l}\text { Moyenne au } \\
\text { temps 02 }\end{array}$ & $3,89(1,45)$ & $4,83(1,54)$ & $4,53(1,66)$ & $4,47(1,00)$ \\
\hline $\begin{array}{l}\text { Moyenne au } \\
\text { temps 03 }\end{array}$ & $4,69(1,62)$ & $4,60(1,55)$ & $4,06(1,77)$ & $4,47(1,30)$ \\
\hline
\end{tabular}

l'infirmier et les infirmières qui ont accepté de participer à l'étude avaient un intérêt ou utilisaient déjà les données probantes dans leur pratique. Le fait qu'ils aient continué à utiliser les données probantes de façon habituelle et régulière pourrait expliquer la similitude des moyennes obtenues lors de l'analyse des résultats. De plus, l'échantillon de l'étude était de 18 participants, donc de petite taille. Dans un échantillon plus élevé, nous aurions pu remarquer une meilleure consistance interne et une plus grande variation dans l'utilisation des données probantes en milieu de soins après l'intervention. Aussi, il faut prendre en considération des facteurs incontrôlables associés aux milieux de soins. Les participants n'ont pas été libérés pour effectuer des lectures ou des recherches bibliographiques sur les données probantes. Ces derniers devaient donc trouver du temps libre hors des heures de travail pour développer de nouvelles habiletés et de nouvelles connaissances en recherche.

Les conditions de travail des infirmières durant la pénurie actuelle et la crise économique du système de santé québécois engendrent une surcharge de travail et un manque de temps chez les infirmières et les infirmiers pour consulter les données de recherches récentes. Incontestablement, le manque de personnel et de ressources financières nuit à l'échange, à la transmission et à l'utilisation des données probantes dans les milieux de soins (O’Donnell, 2003). Cette barrière est clairement démontrée dans diverses recherches effectuées (Hannes et al., 2007; O’Donnell, 2003; Thompson, McCaughan, Cullum, Sheldon \& Raynor, 2005). Les études effectuées sur les barrières à l'utilisation des données probantes affirment qu'un soutien entre collègues puis un temps réservé à la lecture et à l'implantation des pratiques exemplaires sont cruciaux. De ce fait, l'utilisation des résultats probants dans la pratique nécessite un encadrement et un processus d'accompagnement soutenu pour parvenir aux objectifs souhaités (HalabisNassif \& Hatem, 2008; McCormack \& Slater, 2006; Oranta, Routasalo \& Hupli, 2002).

Selon une étude effectuée en 2002 par Oranta, Routasalo et Hupli, les principaux obstacles à l'utilisation de la recherche identifiés par les répondants étaient le fait que la plupart des recherches sont publiées dans une langue étrangère (ici notamment en anglais), que les médecins ne coopèrent pas à la mise en œuvre d'actions concrètes et que les analyses statistiques sont difficiles à comprendre (Oranta et al., 2002). De plus, les cliniciens n'ont pas le temps de développer les habiletés de trouver, de regrouper et d'analyser de façon rigoureuse les résultats de recherches récents et probants et n'ont pas reçu la formation nécessaire à la compréhension et à la critique d'articles de recherches ou de guides de pratiques cliniques (Glacken \& Chaney, 2004; Halabis-Nassif \& Hatem, 2008; Hannes et al., 2007; McCormack \& Slater, 2006; O’Donnell, 2003).

À la suite des analyses effectuées, les auteures ont constaté que peu de facteurs sociodémographiques se sont avérés en lien pour prédire l'utilisation des données probantes de façon quotidienne dans le milieu de travail. Seulement la formation scolaire s'est révélée utile pour prédire l'utilisation des données probantes dans le milieu de travail. D'une part, les infirmières ayant une formation scolaire avancée sont plus sensibilisées à la recherche en soins infirmiers. Dans le programme de formation du baccalauréat, les infirmières sont initiées à la recherche via un cours de 45

\begin{tabular}{|l|c|c|c|}
\hline \multicolumn{4}{|c|}{ Tableau 4. Corrélation entre la somme des temps } \\
\hline & Temps 01 & Temps 02 & Temps 03 \\
\hline Temps 01 & ---- & 0,864 & 0,437 \\
\hline Temps 02 & 0,864 & --- & 0,354 \\
\hline Temps 03 & 0,437 & 0,354 & ---- \\
\hline
\end{tabular}


heures. Ces dernières sont directement plus habilitées à trouver et à utiliser les données des recherches récentes. On retrouve une plus grande réceptivité et une utilisation de résultats de recherche chez les infirmières détenant un baccalauréat ou une maîtrise que chez celles ayant une formation en techniques infirmières (Bonner \& Sando, 2008; Halabis-Nassif \& Hatem, 2008; Koehn \& Lehman, 2008 ; Oranta et al., 2002). Les infirmières cliniciennes spécialisées sont celles qui utilisent le plus souvent les résultats probants dans leur quotidien, car cette exigence est directement reliée à leur fonction et celles-ci disposent de temps et de moyens pour les aider dans cet aspect de leur travail. D'autre part, la spécialisation de l'infirmière est aussi un facteur facilitateur pour intégrer les données probantes dans le travail quotidien. Les infirmières spécialisées développent de plus en plus de connaissances dans un domaine choisi, notamment l'oncologie, afin d'utiliser et de disséminer les données probantes dans les soins aux patients. Pour finir, les années d'expérience d'une infirmière ont également une grande influence sur la façon de dispenser les soins. Les infirmières d'expérience ont un pouvoir d'influence (utilisation persuasive) sur les nouvelles infirmières. Si une infirmière expérimentée effectue son travail en se basant sur les données probantes, il est probable qu'une jeune infirmière pourra s'inspirer de cette dernière pour développer des compétences de travail en utilisant ces données. De plus, il serait judicieux que les responsables du département de soins puissent réviser la charge de travail des infirmières et prendre une décision éclairée au sujet du temps nécessaire pour l'introduction de nouvelles innovations (Halabis-Nassif \& Hatem, 2008).

\section{Formation}

Une recherche effectuée en 2008 par Halabis-Nassif et Hatem donne des conclusions intéressantes. Ces auteurs affirment que le développement de la pratique infirmière fondée sur l'utilisation des résultats probants exige la conjugaison de trois facteurs: une organisation qui soutient le projet, un enseignement approprié de la méthodologie de la recherche et une perception positive des infirmières en regard de la recherche. D'après eux, il serait intéressant de développer les connaissances des infirmières en méthodologie de la recherche et de les accompagner dans leurs premiers travaux. Actuellement, il y a un engouement pour la formation professionnelle continue, la compétence et la démonstration d'une expertise clinique en soins infirmiers. La formation professionnelle continue peut être dispensée sur les unités de soins par une infirmière mentor. Cette dernière est une infirmière ressource attitrée à un poste de mentor, qui met en évidence sa valeur et son utilité au sein d'une organisation de restructuration des connaissances et des pratiques (Whyte, Simon \& Booker, 2007). Les avantages d'un développement professionnel continu et soutenu par une infirmière mentor sont 1) le maintien de normes élevées de soins et de services, 2) l'amélioration et le développement de divers soins et services, 3) l'assurance de la compétence du personnel et 4) la garantie de la responsabilité des actions des praticiens. Bref, le mentor joue un rôle clé dans le transfert des apprentissages cliniques vers la pratique quotidienne (McCormack \& Slater, 2006). La bonne attitude de l'infirmière mentor est un facteur essentiel pour établir et conserver un climat de confiance (Oranta et al., 2002). Par ailleurs, les infirmières mentors peuvent habiliter les infirmières à engendrer des changements qui améliorent les soins aux patients. Elles peuvent également améliorer les conditions de travail en augmentant la satisfaction des employés dans le milieu de travail (Cameron-Buccheri \& Ogier, 1994).

\section{Recherche}

En 2007, (Moriarty, O’Hara \& Byron, 2007), se sont intéressés aux conséquences de l'implantation d'une personne mentor en milieu de soins palliatifs. Le but de cette étude était de décrire et d'évaluer la mise en œuvre de deux postes d'infirmière Macmillan en tant que mentor. Les infirmières Macmillan, qui sont des infirmières de santé communautaire au Royaume-Uni, jouent un rôle significatif dans les services de soins palliatifs, prodiguant aux patients et à leur famille des soins directs ou indirects. Ces professionnelles ont au moins cinq ans d'expérience, dont deux ans ou plus en cancérologie ou en soins palliatifs puis elles ont aussi suivi des cours spécialisés dans la gestion de la douleur et dans le soutien psychologique. Deux infirmières Macmillan ont été employées pour réaliser cette étude. Ces dernières ont été actives dans trois domaines de la pratique soit dans des programmes d'éducation destinés aux infirmières, à la création d'une ressource de soutien et à l'application des lignes directrices en soins palliatifs. Les postes étaient à «double sens »; pour la première partie de la semaine, les détentrices des postes étaient des infirmières en soins palliatifs et pour le reste de la semaine, elles accomplissaient leur rôle d'infirmière mentor en facilitant l'éducation, le soutien et les divers besoins. Ceci dit, les auteurs ne mentionnent pas dans quelle proportion le rôle de mentor était attribué durant la semaine (Moriarty et al., 2007).

Les résultats ont démontré un rehaussement du rôle des intervenants en soins palliatifs au sein de la communauté, à la suite de l'embauche des infirmières mentors. Leur rôle a été très bien accueilli par les professionnels de la santé. Plus précisément, le facteur le plus important identifié pour la mise en œuvre réussie de ces postes a été le "double sens». Le fait que les infirmières aient continué à travailler régulièrement avec l'équipe de travail en soins palliatifs a facilité l'acceptation du nouveau rôle de l'infirmière mentor. Enfin, celles-ci ont apporté des changements majeurs dans les trois domaines où elles ont été actives durant les deux années de l'étude. Par exemple, elles ont formé les infirmières sur divers sujets tels que la douleur, le soutien à apporter aux familles, le matériel d'appoint pour les soins à prodiguer, etc. En plus, elles ont établi une liste de 17 ressources en soins palliatifs que les infirmières peuvent consulter en tout temps afin de perfectionner leurs pratiques. Elles ont aussi implanté dans le milieu les lignes directrices et les données probantes se rattachant aux soins palliatifs. Il s'agit notamment d'un déploiement des résultats probants pour la gestion de la douleur et des symptômes reliés au cancer et aux soins palliatifs. Selon les auteurs, la promotion des lignes directrices cliniques a été retenue comme un indicateur clé de la réussite des postes d'infirmière mentor au sein de l'équipe. Du reste, lorsque les infirmières mentors sont entrées en fonction, il y avait des divergences de pratique à l'égard de l'adoption des lignes directrices pour les soins palliatifs. Les protocoles et techniques de soins n'étaient plus appropriés au point de se révéler désuets. Un certain nombre d'initiatives ont été mises en œuvre pour appliquer une pratique conforme aux lignes directrices et aux dernières données probantes (Moriarty et al., 2007).

Des entrevues avec des médecins, des infirmières et des membres de l'administration ont confirmé que les infirmières Macmillan jouent un rôle clé en soutenant l'adoption de programmes de soins à la fine pointe des connaissances. Selon les infirmières interviewées, les infirmières mentors ont beaucoup apporté au domaine des soins palliatifs en deux ans puisque la qualité des soins a grandement été améliorée pendant que ces dernières étaient en fonction. Finalement, les administrateurs de l'hôpital espèrent que les soins seront désormais basés sur une pratique exemplaire et que les infirmières comprendront davantage le rôle et les services offerts par l'équipe spécialisée en soins palliatifs (Moriarty et al., 2007). Par conséquent, il serait recommandé de poursuivre les recherches sur l'implantation récente du rôle de l'infirmière mentor au Québec et au Canada afin de déterminer son influence sur l'utilisation des données probantes en oncologie (Canadian Partnership Against Cancer: Health Human Resources Action Group, 2009). 


\section{Références}

Adams, L.A., Shepard, N., Caruso, R.A., Norling, M.J., Belansky, H., \& Cunningham, R.S. (2009). Putting evidence into practice: evidence-based interventions to prevent and manage anorexia. Clinical Journal of Oncology Nursing, 13(1), 95-102.

Allard, N. (2005). Day surgery and recovery in women with a suspicious breast lesion: Evaluation of a psychoeducational nursing intervention. Doctoral dissertation: University of Toronto., Toronto.

Association canadienne des infirmières en oncologie (2006). Normes de pratique et compétences pour l'infirmière spécialisée en oncologie. Toronto:ACIO.

Bonner, A., \& Sando J. (2008). Examining the knowledge, attitude and use of research by nurses. Journal of Nursing Management, 16, 334-343.

Burns, N., \& Grove, S.K. (2007). Understanding nursing research: Building an evidence-based practice (4the éd.). St. Louis, Mo.: Saunders Elsevier.

Burns, N., \& Grove, S.K. (2009). The practice of nursing research: Appraisal, synthesis, and generation of evidence (6e éd.). St. Louis, MO: Saunders/Elsevier.

Cameron-Buccheri, R., \& Ogier, M.E. (1994). The USA's nurse managers and UK's ward sisters: Critical roles for empowerment. Journal of Clinical Nursing, 3(4), 205-212.

Canadian Partnership Against Cancer: Health Human Resources Action Group. (2009). E-Mentoring program for oncology APNs: A Pan-Canadian Strategy. Toronto: Authors.

Comité consultatif sur le cancer (1998). Programme québécois de lutte contre le cancer. Québec: Ministère de la Santé et des Services sociaux, Gouvernement du Québec.

Côté, J., \& Pepler, C. (1999). Des stratégies de soins efficaces auprès de personnes vivant avec le VIH. Infirmière du Québec, 7(2), 46-52.

Côté, J.K., \& Pepler, C. (2002). A randomized trial of a cognitive coping intervention for acutely ill HIV-positive men. Nursing Research, 51(4), 237-244.

de Serres, M., \& Beauchesne, N. (2000). L'intervenant pivot en oncologie: un rôle d'évaluation, d'information et de soutien pour le mieux-être des personnes atteintes de cancer: document de réflexion. Québec: MSSS.

Duhamel, P.-G. (2006). Nouvelles approches en oncologie. Nutrition: Science en évolution, 4(2).

Egerod, I., \& Hansen, G.M. (Writers). (2005). Evidence-based practice among Danish cardiac nurses: A national survey. Journal of Advanced Nursing: Blackwell Publishing Limited.

Estabrooks, C. A. (1999). Mapping the research utilization field in nursing. Canadian Journal of Nursing Research, 31(1), 53-72.

Estabrooks, C.A., Floyd, J.A., Scott-Findlay, S., O'Leary, K.A., \& Gushta, M. (Writers). (2003). Individual determinants of research utilization: A systematic review. Journal of Advanced Nursing, 43(5), 506-20.

Fortin, M.-F. (2010). Fondements et étapes du processus de recherche: méthodes quantitatives et qualitatives (2e éd.). Montréal: Chenelière Éducation.

Frasure, J. (2008). Analysis of instruments measuring nurses' attitudes towards research utilization: A systematic review. Journal of Advanced Nursing, 61(1), 5-18. doi: JAN452510.1111/ j.1365-2648.2007.04525.x

Glacken, M., \& Chaney, D. (2004). Perceived barriers and facilitators to implementing research findings in the Irish practice setting. Journal of Clinical Nursing, 13, 731-740.

Goulet, C., Lampron, A., Morin, D., \& Heon, M. (2004). [Evidencebased practice. Part 1: Origins, definitions, critiques, obstacles, advantages and impact]. [Review]. Recherche en soins infirmiers, $76,12-18$.

Goulet, C., Lampron, A., Morin, D., \& Heon, M. (2004). [Evidencebased practice: Part 2: Steps in the process]. [Review]. Recherche en soins infirmiers, 76, 19-29.
Halabis-Nassif, H., \& Hatem, M. (2008). Les résultats probants et le développement d'une culture de recherche infirmière. Perspective infirmière, 5(7).

Hannes, K., Vandersmissen, J., De Blaeser, L., Peeters, G., Goedhuys, J., \& Aertgeerts, B. (2007). Barriers to evidence-based nursing: A focus group study. JAN Original research, 162-171.

Hill, B.J. (1982). Sensory information, behavioral instructions and coping with sensory alteration surgery. Nursing Research, 31, 17-21.

Koehn, M.L., \& Lehman, K. (2008). Nurses' perceptions of evidencebased nursing practice. Journal of Advanced Nursing, 62(2), 209215.

Leventhal, \& Johnson, J.E. (1983). Laboratory and field experimentation: Development of a theory of self-regulation. Dans P. Wooldridge, M. Schmitt, J. Skipper, \& R. Leonard (Éds.), Behavioral science and nursing theory (pp. 189-262). St-Louis: C.V. Mosby Company.

McCormack, B., \& Slater, P. (2006). An evaluation of the role of the clinical education facilitator. Journal of Clinical Nursing, 15.

Miller, M., \& Kearney, N. (Writers). (2004). Chemotherapy-related nausea and vomiting: past reflections, present practice and future management. European Journal of Cancer Care, 13(1), 71-81.

Moore, S.M. (1996). The effects of a discharge information intervention on recovery outcomes following coronary artery bypass surgery. International Journal of Nursing Studies, 33(2), 181-189.

Moriarty, D., O’Hara, A., \& Byron, S. (Writers). (2007). Macmillan nurse facilitators for palliative care: Evaluation of a pilot project. International Journal of Palliative Nursing, 13(7), 334-43.

O'Donnell, C.A. (2003). Attitudes and knowledge of primary care professionals towards evidence-based practice: A postal survey. Journal of Evaluation in Clinical Practice, 10(2), 197-205.

Oncology Nursing Society. (2009a). Patient education. Récupéré de http://www.ons.org

Oncology Nursing Society. (2009b). Updated Oncology Nursing Society putting evidence into practice resources. Clinical Journal of Oncology Nursing, 13(2), 137-143.

Oncology Nursing Society. (2010). ONS PEP ${ }^{\circledR}$-Putting Evidence Into Practice. Récupéré de http://www.ons.org/Research/PEP

Oranta, O., Routasalo, P., \& Hupli, M. (2002). Barriers to and facilitators of research utilization among Finnish Registered Nurses. Journal of Clinical Nursing, 11.

Sackett, D.L., Rosenberg, W.M., Gray, J.A., Haynes, R.B., \& Richardson, W.S. (1996). Evidence-based medicine: What it is and what it isn't. BMJ (Clinical Research Ed.), 312(7023), 71-72.

Santé canada. (2009). Préoccupations liées à la santé: le cancer. Récupéré de http://www.hc-sc.gc.ca/hc-ps/dc-ma/ cancer-fra.php

Société canadienne du cancer. (2010). Statistiques canadiennes sur le cancer 2010. Toronto. Société canadienne du cancer, Institut national du cancer du Canada.

Société canadienne du cancer. (2010). Statistiques québécoises sur le cancer (26 juin 2009). Récupéré de http://www.cancer.ca

Suls, J., \& Wan, C.K. (1989). Effects of sensory and procedural information on coping with stressful medical procedures and pain: A meta analysis. Journal of Counsulting and Clinical Psychology, 57(3), 372-379.

Thompson, C., McCaughan, D., Cullum, N., Sheldon, T., \& Raynor, P. (2005). Barriers to evidence-based practice in primary care nursing-Why viewing decision-making as context is helpful. Journal of Advanced Nursing, 52(4), 432-444.

Vallerand, R.J. (1989). Vers une méthodologie de validation transculturelle de questionnaires psychologiques: implications pour la recherche en langue française. Psychologie canadienne, 30(4), 662-689.

Whyte, D., Simon, A., \& Booker, R. (2007). The role of nurse educators as facilitators for the implementation and evaluation of evidencebased clinical guidelines. Oncology Nursing Forum, 34(2). 